\title{
A predictive mechanical model for evaluating vertebral fracture probability in lumbar spine under different osteoporotic drug therapies
}

\author{
E. López ${ }^{1}$, E. Ibarz ${ }^{2}$, A. Herrera ${ }^{3,4}$, S. Puértolas ${ }^{2}$, S. Gabarre², Y. Más ${ }^{2}$, J. Mateo ${ }^{3,4,5}$, \\ J. Gil-Albarova ${ }^{3,4,5}$ and L. Gracia ${ }^{2}$
}

\section{Highlights}

- Method for estimating the fracture risk in osteoporotic patients based on Damage and Fracture Mechanics and DXA measurements.

- The method evaluates the evolution over time of the mechanical strength of bone from BMD, for different treatments.

- Evolutionary algorithm and a finite element model of the lumbar spine allow the prediction of fracture probability.

- The incorporation of clinical measurements and simulation results will be useful for an individualized treatment in patients.

- The model predicts the evolution of vertebral fracture probability and the expected evolution under different therapies. \footnotetext{
mineral density (BMD) measurements. of damage and fracture probability, identifying high-risk local zones at vertebral body.

${ }^{1}$ Department of Design and Manufacturing Engineering, University of Zaragoza, Spain

${ }^{2}$ Department of Mechanical Engineering, University of Zaragoza, Spain

${ }^{3}$ Department of Surgery, University of Zaragoza

${ }^{4}$ Aragón Health Sciences Institute, Zaragoza, Spain

${ }^{5}$ Department of Orthopaedic Surgery and Traumatology, Miguel Servet University Hospital, Zaragoza, Spain
}

Abstract: Osteoporotic vertebral fractures represent major cause of disability, loss of quality of life and even mortality among the elderly population. Decisions on drug therapy are based on the assessment of risk factors for fracture, from bone

A previously developed model, based on the Damage and Fracture Mechanics, was applied for evaluating the mechanical magnitudes involved in the fracture process from clinical BMD measurements. BMD evolution in untreated patients and in patients with seven different treatments was analyzed from clinical studies, in order to compare the variation in the risk of fracture. The predictive model was applied in a finite element simulation of the whole lumbar spine, obtaining detailed maps

For every vertebra, strontium ranelate exhibits the highest decrease, whereas minimum decrease is achieved with oral ibandronate. All the treatments manifest similar trends for every vertebra. Conversely, for the natural BMD evolution, as bone stiffness decreases, the mechanical damage and fracture probability show a significant increase (as it occurs in the natural history of BMD). Vertebral walls and external areas of vertebral end plates are the zones at greatest risk, in 
coincidence with the typical locations of osteoporotic fractures, characterized by a vertebral crushing due to the collapse of vertebral walls

This methodology could be applied for an individual patient, in order to obtain the trends corresponding to different treatments, identifying at-risk individuals in early stages of osteoporosis and might be helpful for treatment decisions.

Keywords: Osteoporosis, osteoporotic vertebral fracture, predictive model, finite elements, fracture risk, fracture probability, osteoporosis treatments.

\section{Introduction}

Osteoporosis is a systemic skeletal disease characterized by low bone mass and deterioration of bone microstructure, leading to bone fragility and susceptibility to fracture [1]. Currently, osteoporotic fractures are considered as an important public health problem leading to morbidity and mortality, generating very important economic costs [2]. Osteoporosis represents a major cause of disability, loss of quality of life and even death among the elderly population [3-6].

Osteoporosis is caused by a skeletal involution linked to aging, which is more prevalent in women: the lifetime risk for a fragility fracture at the age of 50 lies within the range of $40 \%$ in women [7]. Osteoporosis has been called the silent epidemic because in most cases the first symptom is the appearance of a first fragility fracture, so it is essential to establish treatment to prevent fractures. The decision to treat is based on the analysis of risk factors for fracture.

Vertebral fracture is the most common osteoporotic fracture, being more prevalent in women than in men [8, 9], leading to back pain, kyphosis and severe functional and vital impact in $30-50 \%$ of patients $[10,11]$. Underdiagnosis of osteoporotic vertebral fracture is common for lack of complementary tests in older women who visit the doctor complaining of back pain and sometimes because the symptomatology of the first fracture is not evident $[12,13]$.

Different publications estimate that are diagnosed only $40 \%$ of fractures [12, 14]; other assessments estimate that only $5-20 \%$ of these vertebral fractures are diagnosed in primary care [15]. The first vertebral fracture is a factor of high risk of new fractures, localized in another vertebra or other areas of skeleton [16-20], leading to so-called fracture cascade [21], when new fractures occur in the spine. Decisions on drug therapy in osteoporotic patients without previous fractures are mainly based on the analysis of risk factors which predispose to fracture. A risk assessment tool called FRAX ${ }^{\circledR}$ (Fracture Risk Assessment Tool) has been developed by the World Health Organization (WHO) for this purpose [7, 22-24]. The risk of major osteoporotic fractures (hip, vertebrae, humerus and wrist) over the next 10 years can be estimated with the FRAX ${ }^{\circledR}$ tool. The probability of fracture is calculated on the basis of age, body mass index and several dichotomized variables (previous fracture, smoking, rheumatoid arthritis, etc.). Other studies have questioned the effectiveness of FRAX $^{\circledR}$ as a tool for predicting fracture risk [25-28].

Several previous surveys have assessed the risk of fracture using various methodologies, but mostly based on BMD measurements [29, 30] which is considered to be the most important indicator to diagnose osteoporosis and monitoring treatment [31]. BMD measurements have also been used for determining the mechanical strength [32] or to develop statistical models for predicting the risk of fracture [29, 30, 33-35].

The treatment of osteoporosis includes general measures (breaking harmful habits, proper nutrition, physical exercise and prevention of falls, intake of Calcium and Vitamin D) and pharmacological treatment. The drugs used today are cost-effective [36]. Various medications are available for prevention and treatment of osteoporosis. Pharmacologic interventions preventing fractures in patients with osteoporosis aim at correcting the bone remodeling imbalance by either reducing bone resorption and bone turnover or stimulating bone formation and strontium ranelate both inhibits bone resorption and stimulates bone formation which gives a mixed effect [37, 38]; this last drug is approved in Europe but not in USA. The fundamental aim of pharmacological treatment is to increase BMD, reducing resorption and bone turnover or stimulating bone formation, but not all drugs have the same effect and the increase percentages in BMD are different.

In the field of Finite Element (FE) simulation, different models and methodologies were used for predicting bone strength or fracture risk at different ages and locations. So, Lee et al. (2004) [19] present a micromechanical model of bone behaviour, which is difficult to extrapolate to the scale required to get realistic predictions. Boccaccio et al. (2008) and Zhang et al. (2010) [39, 40] develop more advanced macro-mechanical models, analyzing a complete functional unit of the spine in terms of mechanical behaviour depending on bone density. MacNeil et al. (2012) [41] build a 2D model in the sagittal plane, using bone geometry and BMD measurements obtained from radiographs and Dual-energy X-ray Absorptiometry (DXA), calculating the stiffness in terms of patient's age. For proximal femoral fractures, Kaneko et al. (2011) [42] develop a model based on imaging 
techniques, establishing a statistical correlation between the prediction of bone strength and the risk of osteoporotic fracture. A different methodology is applied by Bryan et al. (2009) [43], who suggest a parametric model incorporating both the geometry and the properties of bone. A similar methodology is used by Bessho et al. (2009) [44], with a parametric analysis concerning load and support conditions. Some authors have begun to incorporate yield criteria for fracture risk prediction, as Derikx et al. (2011) [45] who apply the Drucker-Prager criterion on a model made from Quantitative Computed Tomography (QCT), with asymmetric yielding in tension and compression. In a similar way, Tellache et al. (2009) [46] apply an anisotropic yield criterion on a model constructed from Computed Tomography (CT) for predicting fracture risk. With a different approach, Amin, et al. (2011) [47] performed a comparative analysis of fracture risk predictions based on BMD measurements against those ones obtained from an FE model developed from QCT. Finally, on the matter of drug treatments, Keaveny et al. (2008) [48] analyze the influence on bone strength of Parathyroid Hormone (PTH) and alendronate, using a FE model developed from QCT scans of osteoporotic patients.

Currently, the most popular clinical tool for fracture risk assessment is FRAX $^{\circledR}$, which doesn't consider bone strength as a relevant magnitude. All the aforementioned computational methods use clinical or mechanical magnitudes related to bone fracture in an independent way, without consider their mutual influence as actually happens.

The aim of this work is to apply a numerical model, previously developed [49], for predicting the risk of osteoporotic vertebral fractures based on the Damage Mechanics and Fracture Mechanics, both for the natural BMD evolution and seven different treatments in order to compare the variation in the risk of fracture. The proposed model allows establishing direct relationships between clinical and mechanical magnitudes, so evolution of $\mathrm{BMD}$, bone strength, damage and fracture probability can be simultaneously evaluated at any age form the initial measurements of BMD. It is not intended to replace the models based solely on BMD, but to complement it including mechanical magnitudes involved in the fracturing process, providing more information on the mechanical state of the bone in every analyzed zone at each age.

The novelty of this work compared to previous publications of the authors resides, firstly, in the extension to the whole of the lumbar spine, and on the other hand, in the application of the most common treatments in osteoporotic patients.

\section{Methods}

BMD is the current standard for diagnosis of osteoporosis. Over 120 worldwide published papers assessing lumbar spine BMD evolution, both in natural conditions and in patients under drug therapy, were selected for analysis. Regarding the natural history of BMD, the average curve published by Mazess and Barden (1999) [50] were chosen as a reference. For drug therapy, seven treatments were selected for the comparative study: oral ibandronate (100 $\mathrm{mg} / \mathrm{month}$ ) [51], oral ibandronate (150 $\mathrm{mg} / \mathrm{month})$ [51], intravenous ibandronate (2 mg bimonthly) [52], intravenous ibandronate (3 mg quarterly) [52], strontium ranelate (2 g/day) [53], zoledronic acid (5 mg/year) [54] and Denosumab (60 mg every six months)[55]. These drugs have proven to be effective, and their BMD evolutionary curves show the required regularity during analyzed time period to be used in a numerical simulation.

Bisphosphonates settle in the bone tissue and its effect persists during some time after its administration, and hence it is completely accepted in the clinical practice the use of intermittent or discontinuous treatments. For the ibandronate, several studies with different dosages [51, 52] corroborate its effectiveness, decreasing the risk of vertebral fractures in women with osteoporosis, improving even in week dosage the results obtained with alendronate [56].

Strontium ranelate is an oral drug that enhances preosteoblastic cell replication and osteoblastic differentiation and decreasing abilities of osteoblasts to induce osteoclastogenesis via the calcium sensing receptor and an increase in the OPG/RANKL ratio [57, 58]. Moreover, it decreases bone resorption by inhibition of osteoclast resorbing activity and osteoclastic differentiation [59]. This dual mode of action leads to a rebalance in bone turnover and therefore in an improvement of bone strength [60]. In this case, BMD data are not comparable with others treatments because strontium has an atomic weight higher than calcium, distorting DXA measurements. A correction about 50\% must be done in order to compare with other treatments [61].

Zoledronic acid, a third-generation aminobisphosphonate, slows down bone resorption, allowing the bone-forming cells time to rebuild normal bone architecture and allowing bone remodelling. Its final effect appears as a higher trabecular bone volume and number of trabeculae, lower trabecular separation and higher connectivity among trabeculae. It has shown marked efficacy reducing the risk of vertebral fractures in clinical trials, producing significant reductions in new fractures in postmenopausal women with osteoporosis [54].

Finally, with respect to the use of Denosumab, its action principle deals with the blocking of natural bone resorption. Its effectiveness reducing the fracture risk has 
been demonstrated in different studies a 5 and 8 years studies [62, 63], being recommendable its use in women with a high fracture risk.

As the model evaluates the mechanical strength of bone from BMD, an analytical function expressing its evolution over time, obtained from numerical data contained in the different studies, is needed. Since the standard adjustment techniques (linear or quadratic regression curves) do not provide enough accuracy and reliability to be applied to the predictive model, more complex adjustments have been set out to obtain continuous curves of regression. The evolution trends of the different selected cases in the study can be extrapolated from those continuous curves, ensuring a consistent behaviour. The following adjustments were proposed for the apparent density $\rho$ :

- Polynomial:

$$
\rho=a_{n} t^{n}+a_{n-1} t^{n-1}+\ldots+a_{1} t+a_{0}
$$

- $\quad$ Exponential:

$$
\rho=k\left(1-e^{-\left(a_{n} t^{n}+a_{n-1} t^{n-1}+\ldots+a_{1} t+a_{0}\right)}\right)
$$

- Exponential asymptotic:

$$
\rho=\rho_{0}+\left(\rho_{\text {lim }}-\rho_{0}\right)\left(1-e^{-\frac{a t}{t_{m}-t}}\right)
$$

where $t$ is the age at which apparent density is calculated, $n$ is the polynomial degree and $k$ is an amplitude factor multiplying the normalized exponential function.

These adjustments (Equations 1, 2 and 3) have been applied to the eight examined curves (natural evolution and the seven therapies). These equations apply to different treatments, as detailed in the Results section. Several requirements have been made during this adjustment: the lowest mean square error, the closest to unity correlation coefficient $R^{2}$, and a 10-year standardized follow-up period. That extrapolation is intended to be consistent in mathematical terms, independently of the actual duration of the considered study. Standardization of BMD measurements included in each published paper was required in order to make comparisons among them [64], because the data were obtained from different densitometric equipment depending on the study (Hologic, Lunar and Norland).

In order to assess the different treatments, the predictive model presented in [49] was applied to the different BMD evolutionary curves. As the predictive model takes into account the mechanical parameters involved in the fracture process, a correlation between these magnitudes and those ones measured in clinical terms is firstly required. To this effect, Carter and Hayes (1977) [65] established a direct relationship between Young's modulus, $E$, and bone apparent density, $\rho$, for low strain rates $(0.01)$ :

$$
E=2875 \rho^{3}
$$

On the other hand, the relationship between the BMD value and the apparent density is adjusted, according to BMD clinical measurements and experimental results for apparent density [64], as:

$$
\rho=\rho_{\max }\left(\frac{B M D}{B M D_{\max }}\right)^{\lambda}
$$

being $\lambda$ a numerical parameter that depends on the sample data. For the present study a value of 0.6 was assigned for $\lambda$ in order to fit the actual data presented in [64]; $\rho_{\max }$ is the maximum value for apparent density, $B M D_{\max }$ is the maximum value for BMD and $B M D$ is the actual value for BMD.

The Karganovin's Damage Mechanics model [66] was selected for the simulation of the degenerative process.

A model of fracture probability based on the law of Paris [67], which explains the stable crack growth under monotonic loading, was developed [49]. According to such law, the rate of crack growth (differential relationship between number of stress cycles, $N$, and crack length, $a$ ) is expressed as:

$$
d N=\frac{d a}{\alpha(\Delta \sigma)^{\beta} \delta^{\beta / 2} a^{\beta / 2}}
$$

By integrating equation (6), the number of loading cycles, $N_{i-c}$, needed to increase mechanical damage from the $D_{i}$ value to the $D_{c}$ value was expressed as follows:

$$
N_{i-c}=\frac{\left[\left(\frac{1}{\omega D_{i}}\right)^{\frac{\beta}{2}-1}-\left(\frac{1}{\omega D_{c}}\right)^{\frac{\beta}{2}-1}\right]}{\alpha(\Delta \sigma)^{\beta} \delta^{\beta / 2}\left(\frac{\beta}{2}-1\right)}
$$

where $\alpha$ and $\beta$ are material parameters associated with the law of Paris [67], $\delta$ is a parameter related to the stress intensity factor defined in Linear Elastic Fracture Mechanics, which depends on geometric aspects and stress distribution, $\Delta \sigma$ is the average variation of stress in each loading cycle and $\omega$ is a parameter which relates the mechanical damage to the crack size $a(a=\omega D)$.

By normalizing the probability of fracture, assigning value 1 to critical damage $\left(D=D_{c r i}\right)$ and value 0 to no damage $(D=0)$, and assuming that the average variation of stress in each loading cycle remains constant, we finally 
obtain the probability of fracture as a function of the initial, $D_{i}$, the current, $D_{c}$, and the critical, $D_{c r i}$, damages:

$$
P\left(D_{c}\right)=\frac{\left(\frac{1}{D_{i}}\right)^{\frac{\beta}{2}-1}-\left(\frac{1}{D_{c}}\right)^{\frac{\beta}{2}-1}}{\left(\frac{1}{D_{i}}\right)^{\frac{\beta}{2}-1}-\left(\frac{1}{D_{c r i}}\right)^{\frac{\beta}{2}-1}}
$$

In the above expression, $D_{i}$ is the damage corresponding to the mechanical situation of the bone at the age of the patient and $D_{c}$ is the estimated damage for the time at which fracture probability is evaluated. If no initial damage, $D_{i}$, is detected, the probability is calculated as:

$$
P\left(D_{c}\right)=\left(\frac{D_{c}}{D_{c r i}}\right)^{\frac{\beta}{2}-1}
$$

Initial damage, $D_{i}$, is obtained considering the mechanical properties corresponding to the actual age of the patient. In this way, only mechanical damage $(D)$ and $\beta$ coefficient are needed in order to calculate the fracture probability.

A value 5 has been given to the $\beta$ coefficient for cortical bone, according to Taylor [68] , and, in accordance with Kargarnovin et al. [66], a critical damage, $D_{c r i}$, of 0.38 and a critical strain, $\varepsilon_{c r i}$, of 0.0174 have been considered, with a variable strain threshold, $\varepsilon_{0}$, according to age (Figure 1) with a variation equivalent to the corresponding to BMD evolution (no damage is produced by strains below strain threshold).

BMD evolutionary curves for a specific population are basic references and provide only general information. In order to apply the model to specific patients we must consider, in addition to the trend, the reference density value measured for the patient. In accordance with the law of interpolated natural evolution, the density matching with the age of the patient, $\rho_{p}^{N}(t)$, is given by:

$$
\rho_{p}^{N}(t)=\rho_{N}(t)+\left[\rho_{0}-\rho_{N}\left(t_{0}\right)\right]
$$

being $\rho_{N}\left(t_{0}\right)$ the average density measured in the patient and $\rho_{0}$ the matching value from the reference progression curve, that is, the actual patient's density that may be different to the average curve considered for the whole population. In fact, there is an offset that should be added to the reference evolutionary curve of density, providing a translation of the average curve, allowing an adaptation for each individual patient. 

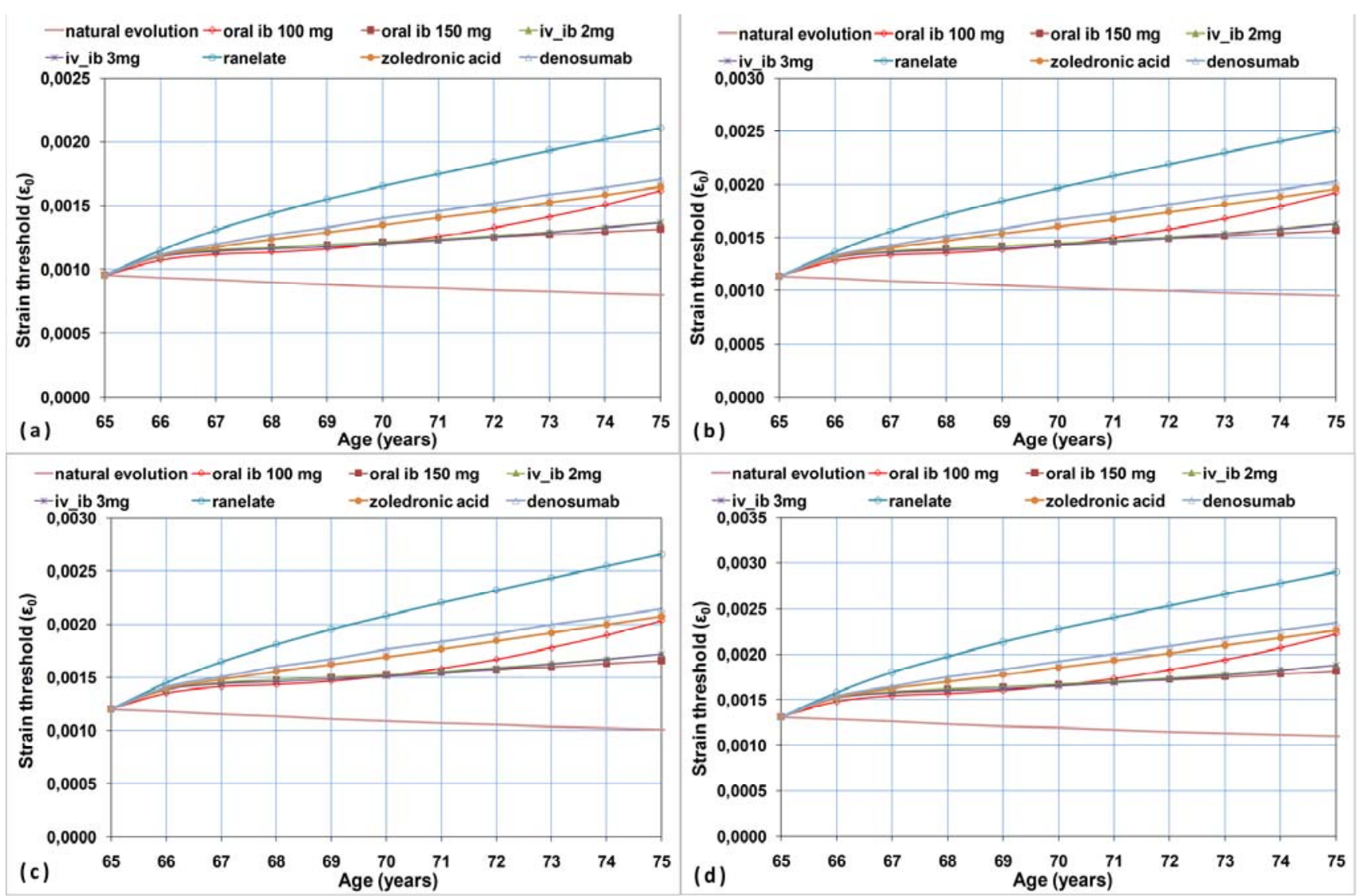

Figure 1 - Evolutionary curves for strain threshold (natural evolution and the seven treatments): a) L1; b) L2; c) L3; d) L4

When a drug therapy is applied to the same patient, a similar correction is required because a new offset appears. So, the progression curve for this patient under a treatment would be:

$$
\rho_{p}^{T}(t)=\rho_{T}(t)+\left[\rho_{0}-\rho_{T}\left(t_{0}\right)\right]
$$

In Equations 10 and 11, the subscripts or superscripts $N$ and $T$ represent natural evolution or evolution with treatment, respectively. All these adjustments provide the estimated BMD value for a specific patient at any age and under any prescribed therapy. From this value, mechanical properties of bone can be calculated. It must be noticed that the considered curves represent the mean evolutionary curves for the population, and an individual patient could not follow the curve exactly but in an approximate way.

Densitometric data of the healthy lumbar spine have been taken as the starting point for this study, based on previous published work [50]. As only information corresponding to $2 \mathrm{D}$ average values was available from

BMD measurements for every vertebra and the vertebra includes five zones with different apparent density and bone properties as it is well known [69], an algorithm was implemented in order to assign the values corresponding to every zone. Firstly a mean value was defined for the apparent density by accounting the volume of every zone with its corresponding density (i.e., addition of the product of volume times density divided by the total volume). Then that mean value was obtained from equation (5) for every vertebra. Finally, performing the inverse process specific values of BMD apparent density and Young modulus were assigned to each of the five zones (Table 1). This procedure is repeated along the evolutionary process.

From all the previous calculations, an evolutionary algorithm has been implemented (Figure 2), which has been used combined with a finite element model of the spine containing T12-S1 segments (Figure 3), obtained from a previous model of the lumbar spine [70]. T12-L5 vertebrae and S1 reproduce in a reliable way the anatomy of spine. 
Table 1 - BMD, apparent density and Young's modulus for the data corresponding to the study of López et al. (2010)[64].

\begin{tabular}{cccc}
\hline Zone & $\begin{array}{c}\text { Standard } \\
\text { BMD } \\
\left(\mathbf{m g} / \mathbf{c m}^{2}\right)\end{array}$ & $\begin{array}{c}\text { Apparent } \\
\text { volumetric } \\
\text { density } \\
\left(\mathbf{g r} / \mathbf{c m}^{\mathbf{3}}\right)\end{array}$ & $\begin{array}{c}\text { Young } \\
\text { modulus } \\
\mathbf{( M P a )}\end{array}$ \\
\hline $\begin{array}{c}\text { Vertebral } \\
\text { wall }\end{array}$ & 1159 & 1.494 & 9593 \\
\hline $\begin{array}{c}\text { Outer } \\
\text { vertebral } \\
\text { endplates }\end{array}$ & 1159 & 1.494 & 9593 \\
\hline $\begin{array}{c}\text { Intermediate } \\
\text { vertebral } \\
\text { endplates }\end{array}$ & 610 & 1.186 & 4797 \\
\hline $\begin{array}{c}\text { Centre of } \\
\text { vertebral } \\
\text { endplates }\end{array}$ & 221 & 0.822 & 1599 \\
\hline Apophyses & 321 & 0.941 & 2398 \\
\hline
\end{tabular}

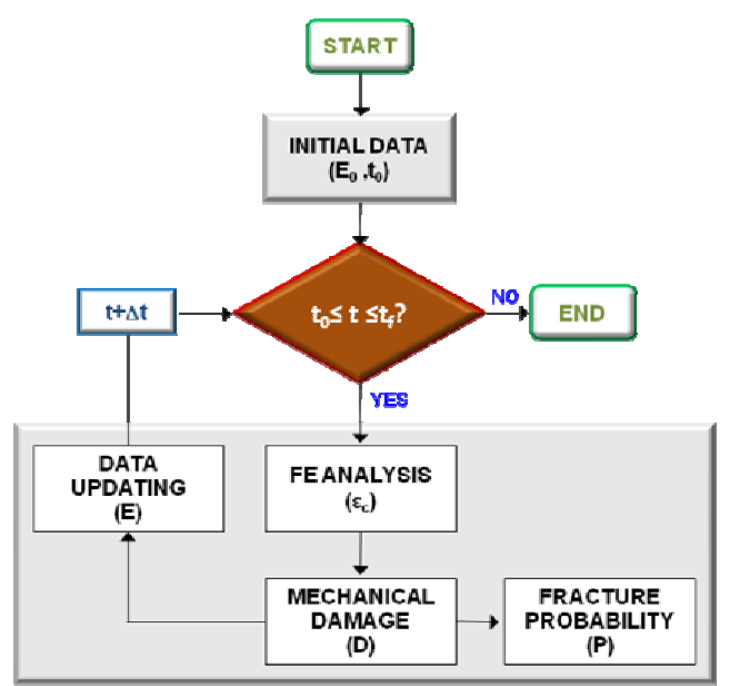

Figure 2 - Evolutionary algorithm for the prediction of fracture probability 


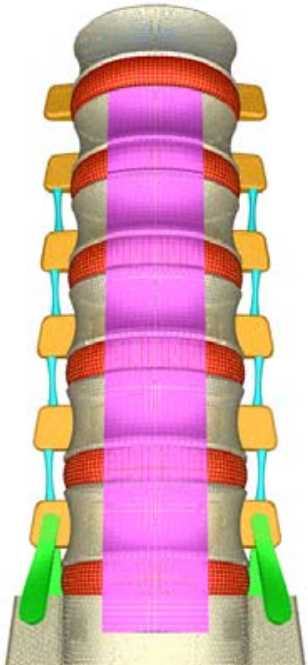

(a)

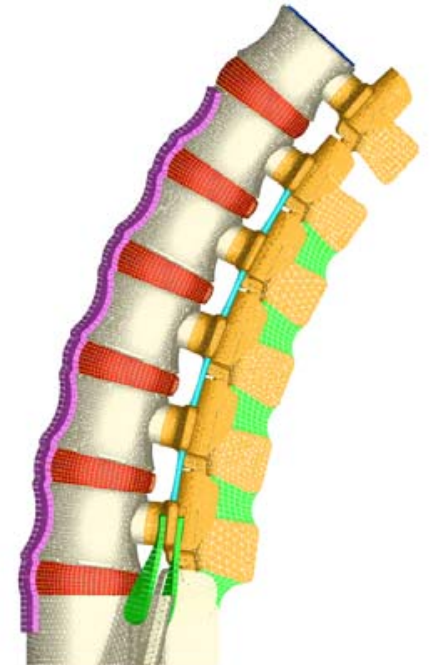

(b)

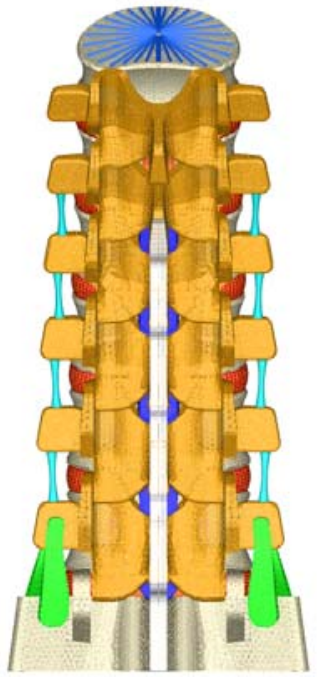

(c)

Figure 3 - Complete FE model including vertebrae, discs and ligaments: a) Frontal view; b) Lateral view; c) Dorsal view

The vertebrae were meshed by means of tetrahedral elements with quadratic approximation in the I-DEAS ${ }^{\circledR}$ program[71] as described in [70]. The mesh of the discs is essential for the correct reproduction of the biomechanical behaviour of the lumbar spine; in order to do this, each disc is divided into nucleus pulposus and annulus fibrosus with commonly accepted dimensions [72]. Each part is meshed separately, the nucleus by means of tetrahedra and the annulus by means of hexahedra and prisms with quadratic approximation. Later, nine layers (outer and four double crossed) of concentric fibres are added to the annulus. These layers are modelled by means of tensiononly elements, included in the hexahedra matrix, with variable orientation from the most internal to the most external (Figure 4), ranging from $35^{\circ}$ to $80^{\circ}$, respecting at most the anatomical disposition [73].

Finally, the ligaments are modelled by means of tetrahedra and prisms with quadratic approximation; in addition, membrane elements have been used for capsular ligaments. The dimensions of those soft tissues correspond to average anatomical measurements $[72,73]$. The number of finite elements for every part is shown in Table 2. The total number of elements of the final mesh, obtained after a sensitivity analysis, is 447462 . To this respect a mesh refinement was performed in order to achieve a convergence towards a minimum of the potential energy, both for the whole model and for each of its components, with a tolerance of $1 \%$ between consecutive meshes.
The bone and ligament properties were taken from the bibliography. Concerning the bone, in [69] it is demonstrated that the centre of the vertebrae is less rigid than in the exterior zone. For this reason the vertebrae are divided into four areas with variable modulus of elasticity (Figure 5). In addition, the corresponding properties are used for the cancellous bone. Material properties are included in Table 2.

The behaviour of the nucleus pulposus, like a noncompressible fluid, was simulated by means of the hyperelastic Mooney-Rivlin model (incompressible) incorporated in the Abaqus ${ }^{\circledR}$ version 6.12 materials library [74]. The fibres of the annulus exhibit a non-linear only tension behaviour approximated using different linear models for each layer considering their respective range of deformation [69]. The materials of the matrix and cartilage of the apophyses were simulated as elastic materials. Finally, the different ligaments present nonlinear only tension behaviour, included as a bilinear model in the strain range (Table 2) as with most of the reported FEM studies [69, 70].

As boundary conditions displacements in the wings of sacrum have been prevented (Figure 6(a)). As load condition a moment of $15 \mathrm{mN}$ was considered acting on the upper face of vertebra T12 (Figure 6(b)), corresponding to the load producing the movement of flexion [70]. 


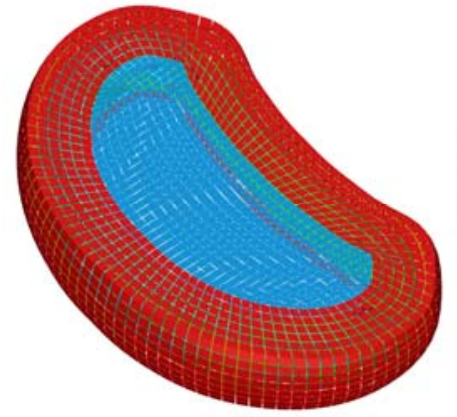

(a)

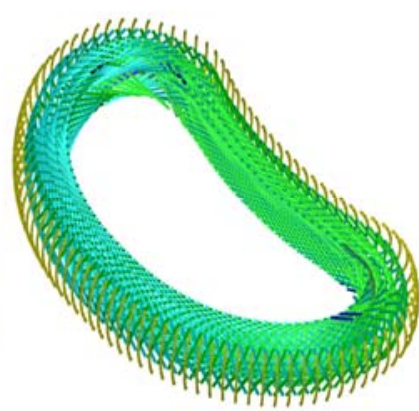

(b)

Figure 4 - Model of the inter-vertebral disk and its layers of fibres: a) Mesh of nucleus pulposus and annulus fibrosus; b) Mesh of fiber layers
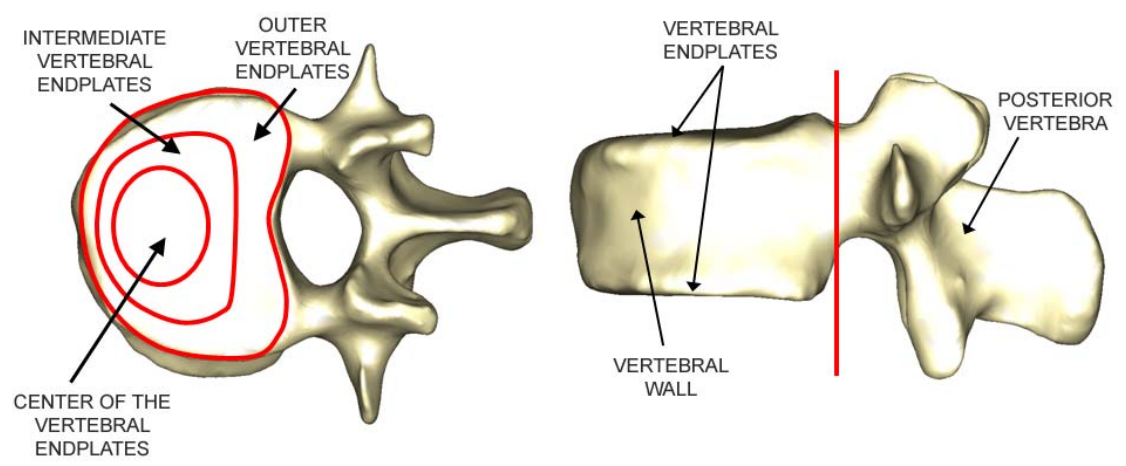

Figure 5 - Zones of different elastic properties in the vertebral body
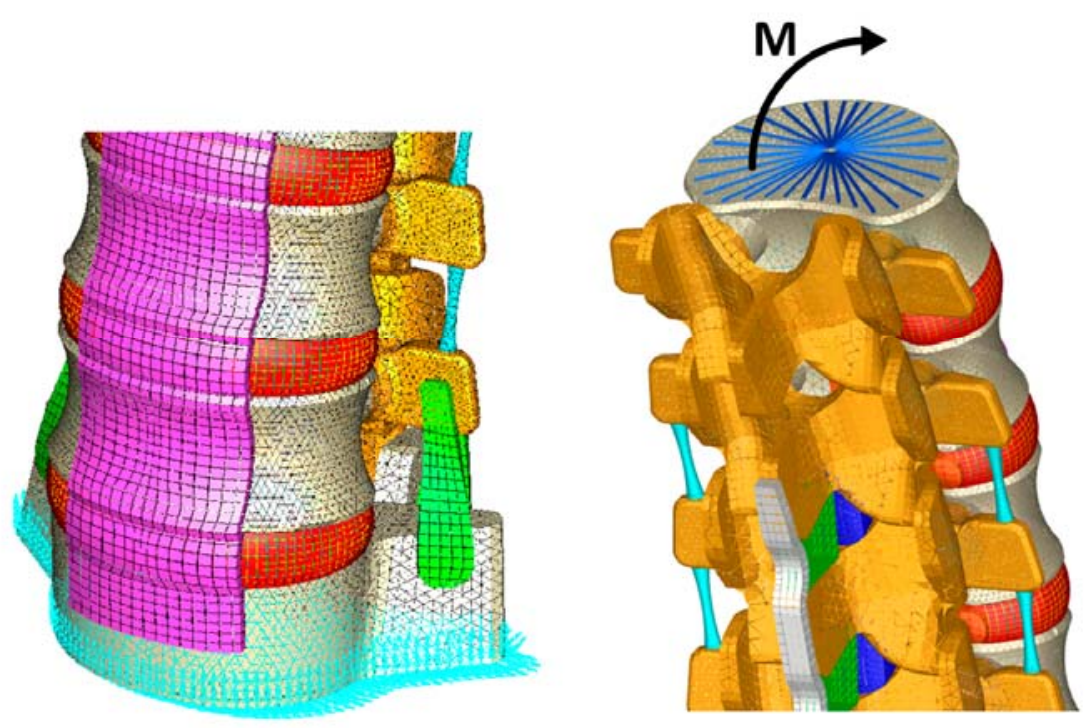

Figure 6 - Boundary conditions: a) Restrained displacements; b) Loads on T12 
Table 2 - Statistics for the FE model and mechanical properties of materials, for healthy lumbar spine, according with [70][Ibarz et al. (2013)]

\begin{tabular}{|c|c|c|c|c|}
\hline Material & $\begin{array}{c}\text { Young } \\
\text { modulus } \\
(\mathrm{MPa})\end{array}$ & $\begin{array}{c}\text { Poisson } \\
\text { coefficient }\end{array}$ & Element type & $\begin{array}{c}\text { Number of } \\
\text { elements }\end{array}$ \\
\hline Outer vertebral endplates & 12000 & 0.3 & Tetrahedron & 3578 \\
\hline Intermediate vertebral endplates & 6000 & 0.3 & Tetrahedron & 2244 \\
\hline Centre of the vertebral endplates & 2000 & 0.3 & Tetrahedron & 831 \\
\hline Walls of the vertebral body & 12000 & 0.3 & Tetrahedron & 37205 \\
\hline Cancellous bone (inside vertebrae) & 100 & 0.2 & Tetrahedron & 44954 \\
\hline Posterior vertebra & 3000 & 0.3 & Tetrahedron & 47134 \\
\hline Cartilage & 50 & 0.4 & Wedge & 3086 \\
\hline Annulus fibrosus & 4.2 & 0.45 & Hexahedron & 8288 \\
\hline Nucleus pulposus $\left(^{*}\right)$ & \multicolumn{2}{|c|}{ Incompressible material } & Tetrahedron & 14410 \\
\hline Annulus fiber layers 1 & 360 & 0.3 & Truss $(* *)$ & 592 \\
\hline Annulus fiber layers 2 & 408 & 0.3 & Truss $(* *)$ & 592 \\
\hline Annulus fiber layers 3 & 455 & 0.3 & Truss $(* *)$ & 592 \\
\hline Annulus fiber layers 4 & 503 & 0.3 & Truss $(* *)$ & 592 \\
\hline Annulus fiber layers 5 & 550 & 0.3 & Truss $(* *)$ & 296 \\
\hline Ligament & $\begin{array}{c}\text { Young } \\
\text { modulus } \\
(\mathrm{MPa})\end{array}$ & $\begin{array}{l}\text { Transition } \\
\text { strain (\%) }\end{array}$ & Element type & $\begin{array}{l}\text { Number of } \\
\text { elements }\end{array}$ \\
\hline \multirow[t]{2}{*}{ Anterior longitudinal ligament } & 7.8 & 12.0 & Wedge $(* *)$ & 9046 \\
\hline & 20.0 & & & \\
\hline \multirow[t]{2}{*}{ Posterior longitudinal ligament } & 10.0 & 11.0 & Wedge $(* *)$ & 3844 \\
\hline & 50.0 & & & \\
\hline \multirow[t]{2}{*}{ Ligamentum flavum } & 15.0 & 6.2 & Tetrahedron $(* *)$ & 3042 \\
\hline & 19.0 & & & \\
\hline \multirow[t]{2}{*}{ Intertransverse ligament } & 10.0 & 18.0 & Tetrahedron $(* *)$ & 6678 \\
\hline & 59.0 & & & \\
\hline \multirow[t]{2}{*}{ Capsular ligament } & 7.5 & 25.0 & Membrane $(* *)$ & 3220 \\
\hline & 33.0 & & & \\
\hline \multirow[t]{2}{*}{ Interspinous ligament } & 8.0 & 20.0 & Tetrahedron $(* *)$ & 2856 \\
\hline & 15.0 & & & \\
\hline \multirow[t]{2}{*}{ Supraspinous ligament } & 10.0 & 14.0 & Tetrahedron $(* *)$ & 2657 \\
\hline & 12.0 & & & \\
\hline \multirow[t]{2}{*}{ Iliolumbar ligament } & 7.8 & 12.0 & Wedge $(* *)$ & 816 \\
\hline & 20.0 & & & \\
\hline
\end{tabular}

(*) $\mathrm{C}_{01}=0.0343 \mathrm{MPa} ; \mathrm{C}_{10}=0.1369 \mathrm{MPa}$. An elastic analysis with Young modulus of $1.0 \mathrm{MPa}$ and Poisson ratio of 0.49 was carried out with similar results and a volume change less than $0.6 \%$.

$(* *)$ Only tension 
The model has been used in predicting the evolution of vertebral fracture probability, by comparing the natural history and the expected evolution under different therapies. The study has been performed for a 65 years old patient with actual BMD values of $718 \mathrm{mg} / \mathrm{cm}^{2}$ for $\mathrm{L} 1,803 \mathrm{mg} / \mathrm{cm}^{2}$ for L2, $834 \mathrm{mg} / \mathrm{cm}^{2}$ for L3 and 883 $\mathrm{mg} / \mathrm{cm}^{2}$ for $\mathrm{L} 4$, respectively. The period of study was ten years (from 65 to 75 years old).

\section{Results}

Firstly, adjustment models were applied both to BMD physiological curve (weighted average from [50] and the curves of patients under seven different therapies: oral ibandronate (100 mg/month) [51], oral ibandronate (150 $\mathrm{mg} / \mathrm{month}$ ) [51], intravenous ibandronate (2 mg bimonthly) [52], intravenous ibandronate (3 mg quarterly) [52], strontium ranelate (2 g/day) [53], zoledronic acid (5 mg/year) [54] and Denosumab (60 mg every six months) [55]. Figure 7 shows the different evolutionary curves obtained in each case, together with the interpolated curves for L1 vertebra. Despite the irregularity of some cases, the correlation coefficients have been 0.994 for natural BMD evolution curve, and in the range 0.9920.999 for BMD evolution curves corresponding to the different treatments. Similar curves were obtained for L2, L3 and L4 vertebrae, respectively.

As can be seen in the graphics, patient treated with oral ibandronate showed a significant initial increase in bone density, more pronounced for $150 \mathrm{mg} / \mathrm{month}$ dose, which remained stable and with increasing trend until the end of the study period. The same evolution happens in the case of intravenous ibandronate. Strontium ranelate also leads to a remarkable increase in BMD during the study period; however, a more continuous increasing is observed, with less initial increments but with higher final values of BMD. Similar trends are observed for zoledronic acid and Denosumab, with a more continuous increasing in the last case. In opposition, the physiological curve (natural evolution) was characterized by a substantial and progressive decline in BMD. For L2, L3 and L4 vertebrae the same tendencies are obtained, starting with the corresponding BMD values.

Figures 8(a), 8(c), 8(e) and 8(g) illustrate the evolution of average mechanical damage under natural conditions and under the seven proposed therapies for L1, L2, L3 and L4 vertebrae, respectively. In the case of therapies which preserve BMD, and therefore bone stiffness, the mechanical damage value diminishes for every treatment. For every vertebra, strontium ranelate exhibits the highest decrease, whereas minimum decrease is achieved with oral ibandronate (150 mg/month). All the treatments manifest similar trends for every vertebra. Conversely to previous trends, for the natural BMD evolution, as bone stiffness decreases, the mechanical damage shows a significant increase (as it occurs in the natural history of BMD).

As a final result, the evolutionary curves of fracture probability were obtained from the evolution of mechanical damage (Fig. 8). As can be seen, the fracture probability shows a marked increase in the natural evolution curve, ranging from a maximum of $5.53 \%$ for L1 to a minimum of $2.06 \%$ for L4. On the other hand, the curves of the treated patient show lower degrees of risk, depending on the therapy type. Thus, for L1 strontium ranelate produces a maximum fracture probability reduction of $16.20 \%$, while oral ibandronate (150 $\mathrm{mg} /$ month) produces a minimum fracture probability reduction of $8.53 \%$. Similarly, for L2 strontium ranelate produces a maximum fracture probability reduction of $5.85 \%$, while oral ibandronate (150 mg/month) produces a minimum fracture probability reduction of $4.00 \%$. For L3 strontium ranelate produces a maximum fracture probability reduction of $4.65 \%$, while oral ibandronate (150 mg/month) produces a minimum fracture probability reduction of $3.22 \%$. Finally, For L4 strontium ranelate produces a maximum fracture probability reduction of $3.09 \%$, while oral ibandronate (150 mg/month) produces a minimum fracture probability reduction of $2.23 \%$. In the best case, an improvement (fracture probability reduction) of $21.73 \%$ between natural evolution and treatment is obtained for L1.

In addition to previous results, programmed subroutines make it possible to obtain damage and fracture probability maps and to identify high-risk zones of the vertebral body. Vertebral walls and external areas of vertebral end plates are the zones at greatest risk, in coincidence with the typical locations of osteoporotic fractures, characterized by a vertebral crushing due to the collapse of vertebral walls. It must be pointed out that the fracture probability maps doesn't represent actual fracture zones, but zones with poorer bone mechanical strength due to cumulative damage. The actual fracture zone would be determined according loading scenario (impact, accidental loads, etc.). 

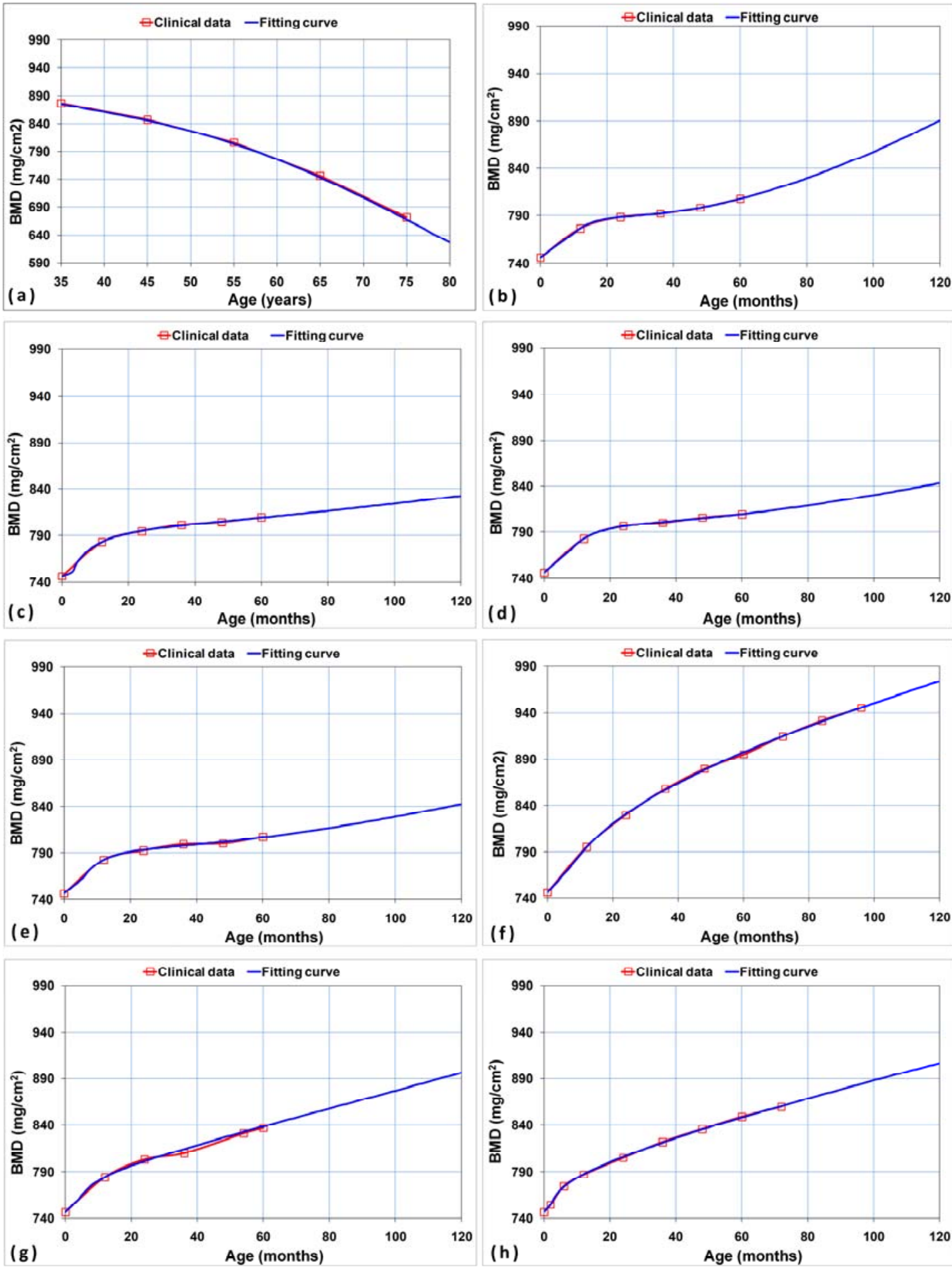

Figure 7 - Fitting of BMD evolutionary curves: a) Natural evolution (polynomial, $\left.\mathrm{R}^{2}=0.994\right)$ ) b) oral ibandronate (100 $\mathrm{mg} / \mathrm{month}$ ) (exponential, $\mathrm{R}^{2}=0.999$ ); c) oral ibandronate (150 mg/month) (exponential, $\mathrm{R}^{2}=0.999$ ); d) intravenous ibandronate ( $2 \mathrm{mg}$ bimonthly) (exponential, $\mathrm{R}^{2}=0.999$ ); e) intravenous ibandronate (3 mg quarterly) (exponential, $\left.\mathrm{R}^{2}=0.998\right) ;$ f) strontium ranelate (2 g/day) (exponential, $\left.\mathrm{R}^{2}=0.999\right)$; g) zoledronic acid (5 mg/year) (exponential, $\left.\mathrm{R}^{2}=0.992\right)$; h) Denosumab (60 mg/6 months) (exponential, $\mathrm{R}^{2}=0.999$ ) 

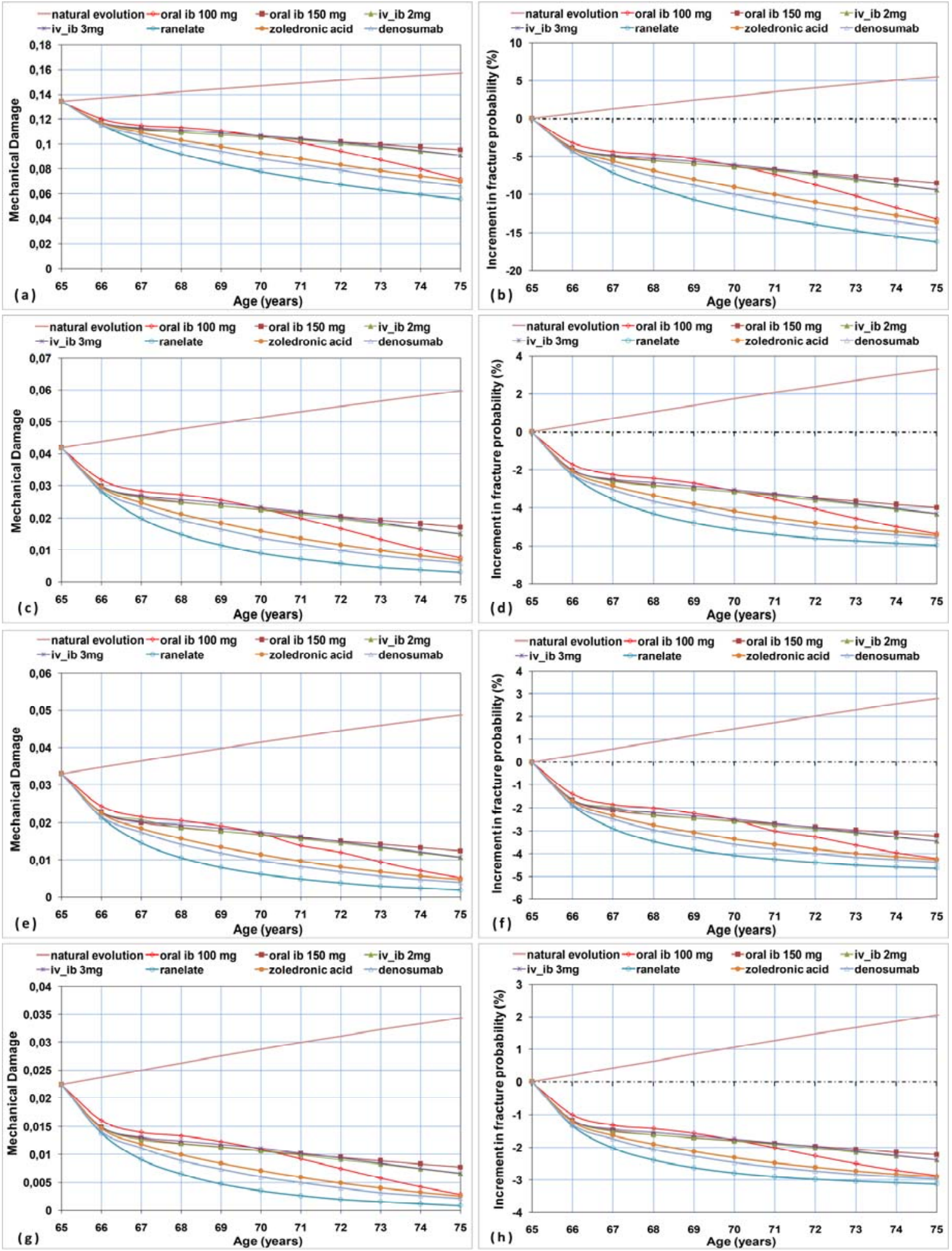

Figure 8 - Evolution of mechanical damage and fracture probability increment for natural BMD evolution and for the different treatments: a) Mechanical damage (L1); b) Fracture probability increment (L1); c) Mechanical damage (L2); d) Fracture probability increment (L2); e) Mechanical damage (L3); f) Fracture probability increment (L3); g) Mechanical damage (L4); h) Fracture probability increment (L4) 


\section{Discussion}

The spinal localization of osteoporotic fractures are very common [75] and unfortunately many of these fractures are usually not diagnosed [12, 13]. It is essential to prevent the first vertebral fracture and therefore determine the risk of fracture is an important aid to the medical doctors for taking decision to prescribe treatment; so the availability of a tool to inform about of individualized fracture risk is very important.

In this work, clinical data (BMD measures) and mechanical magnitudes related to bone strength have been combined in this tool for estimating the risk in osteoporotic patients. The mechanical properties of bone are updated from BMD values obtained from clinical data of untreated patients and in those under different treatments. The model uses Damage and Fracture Mechanics concepts to evaluate the fracture probability in an evolutionary algorithm and can be used in a personalized way from BMD measurements in each case. Many studies have been carried out, both in the clinical $[14-18,20,21,23,76]$ and the simulation fields [19, 28$30,32,34,39,40,77]$. But a simple and reliable model, useful as a tool for diagnosis and prevention in our daily practice, has not yet been achieved. The proposed model can contribute to the development of diagnostic tools for detection of early stages of osteoporosis. It may also be helpful for treatment decisions in selected patients.

There are few studies, besides the tool $\mathrm{FRAX}^{\circledR}$, to determine the risk of vertebral fracture. Some studies assess the risk of new fracture after the diagnosis of first vertebral fracture by using clinical data [15]. Other studied employed logistic regression models based in clinical data and BMD to determine the vertebral fracture risk [78]. Statistical models are currently the most reliable in the literature $[13,14,18,76]$, regardless of mechanical issues involving bone strength estimated for different conditions and ages. The development of new techniques for measuring BMD has focused much of the recent research in the clinical setting, but the mechanical aspects have not been adequately studied [16, 17, 20, 21]. Only in Kanis et al. [79] a new method derived from DXA measures was developed for bone strength assessment.

Concerning FE simulation, the incorporation of the latest imaging techniques (QCT) and BMD measurement (DXA) [19, 29, 39, 77] has allowed the improvement of these models. However, most of models don't consider essential aspects in fracture analysis. Nonetheless, all models assume that bone mineral density is the basic measurement, and it should therefore be used as a benchmark in predicting fracture risk.

From a mechanical point of view, the exposition of the bone to cyclic loads of high value in a damaged bone, once the degenerative process is started, decreases its strength over the time and produces a cumulative damage which can lead to a final fracture. It seems apparent that Damage Mechanics and Fracture Mechanics criteria should be incorporated in any model intending to obtain reliable results. In this regard, the model combines all these requirements. The developed model takes into account the above criteria and combines information of clinical and mechanical magnitudes, providing more complete information about the risk of fracture in the mid and long term, according to the different medical treatments.

Any case, the predictions of the proposed method, based on BMD measurements and mechanical magnitudes are consistent with the clinical models for predicting osteoporotic fractures. In a previous work published by the authors [80], the good correlation between predictions of the proposed method and the FRAX was demonstrated in application to the femur, for a sample size of 220 patients of both sexes aged between 60 and 89 years.

The model presents some limitations, first, as it is well know that life style and changes in habits can affect $\mathrm{BMD}$, so the supposition of crack growth under monotonic loading adopted in the model may be a suitable approximation to predict evolution in older people with osteoporosis, but not in people with variation in their life habits where range and type of load cycles change continuously. In the second term, a more accurate bone density distribution by individual elements in the mesh could be used, planning a complete collection of BMD data, by means of DXA or CT scan images. More complex mechanical damage models could be incorporated taken as basis the model shown, including mechanical behaviour of anisotropic or mixed models. It could also be possible to include crack growth models fitting to the results from in vitro bone fracture. Despite DXA measurements just quantify bone mass and not bone quality, it is widely accepted as a macroscopic indicator of bone strength and stiffness and also that microfractures exert an important influence on the mechanical strength of the bone. In this respect, the influence of trabecular architecture (bone quality) should be considered in the model. On the other hand, patientspecific models are needed [81], and the effects of intervertebral discs should be considered [82], due to the influence of the vertebral geometry and the mechanical properties of discs in the biomechanical behaviour of the whole lumbar spine. These topics should be addressed in future studies. Finally, clinical trials are needed to validate the proposed model in order to apply it to the clinical practice helping for treatment decisions.

Since this is a first application to the complete lumbar spine, we used for simplicity a single model. However, the methodology for the adjustment of the model to specific patients has been developed and it has been successfully tested in previous studies [80]. 
The use of BMD measurements from DXA as input data in the model is due to it is the most commonly available clinical technique. Obviously, more complete data obtained through more powerful techniques (3D scanner, for example), would improve the reliability of the method.

\section{Conclusions}

A mechanical model based on Damage and Fracture Mechanics and DXA measurements, for predicting the probability of fracture in osteoporotic patients has been applied to seven different treatments, identifying the reduction achieved for the fracture probability in every case. The model represents a first step towards the development of new tools for prediction of fracture risk. The incorporation of clinical measurements and simulation results will be useful for an individualized monitoring and treatment in specific patients.

The developed model is not intended to replace the models based solely on BMD, but to complement it including mechanical magnitudes involved in the fracturing process, providing more information on the mechanical state of the bone in every analyzed age.

\section{List of abbreviations}

BMD: Bone Mineral Density

CT: Computed Tomography

DXA: Dual-energy X-ray Absorptiometry

FE: Finite Elements

FRAX: Fracture Risk Assessment Tool

L1 to L5: Lumbar vertebrae

PTH: Parathyroid Hormone

QCT: Quantitative Computed Tomography

S1: Sacrum

T12: Last thoracic vertebrae

WHO: World Health Organization

\section{Competing interests}

The authors declare that they have no conflict of interest.

\section{Authors' contributions}

AH and LG conceived the approach of this work. EL and LG conceived and developed the predictive model for fracture probability. AH, JG-A and JM contribute with clinical measurements and experience with osteoporotic patients. EI, SP, SG and YM conceived and developed the finite element model and carried out all the simulations. $\mathrm{AH}$ and LG coordinated the work between surgeons and engineers. All authors participated in the drawing up of the manuscript, and read and approved the final manuscript.

\section{Acknowledgements}

This work has been partially developed as part of the research project "Development and Clinical Application of a Mechanical Model for the Analysis of Spine Fractures due to Low Energy Trauma, based on Bone Mineral Density Measurements”, supported by Mutua Madrileña Foundation.

\section{References}

1. Peck, W.A., et al., Consensus Development Conference - Diagnosis, Prophylaxis, and Treatment of Osteoporosis. American Journal of Medicine, 1993. 94(6): p. 646-650.

2. Gabriel, S.E., et al., Direct medical costs attributable to osteoporotic fractures. Osteoporos Int, 2002. 13(4): p. 323-30.

3. Kuehn, B.M., Better osteoporosis management a priority - Impact predicted to soar with aging population. Jama-Journal of the American Medical Association, 2005. 293(20): p. 2453-8.

4. Foundation, N.O., Clinician's Guide to Prevention and Treatment of Osteoporosis. 2010: National Osteoporosis Foundation, Washington DC, USA.

5. Reginster, J.Y. and N. Burlet, Osteoporosis: a still increasing prevalence. Bone, 2006. 38(2 Suppl 1): p. S49.

6. Sambrook, P., Cooper, C. , Osteoporosis. 2006, The Lancet. p. 2010-2018.

7. Johnell, O. and J. Kanis, Epidemiology of osteoporotic fractures. Osteoporos Int, 2005. 16 Suppl 2: p. S3-7.

8. Cooper, C., T. O'Neill, and A. Silman, The epidemiology of vertebral fractures. European Vertebral Osteoporosis Study Group. Bone, 1993. 14 Suppl 1: p. S89-97.

9. Wasnich, R.D., Vertebral fracture epidemiology. Bone, 1996. 18(3 Suppl): p. 179S-183S.

10. Kado, D.M., et al., Vertebral fractures and mortality in older women: a prospective study. Study of Osteoporotic Fractures Research Group. Arch Intern Med, 1999. 159(11): p. 1215-20.

11. Nevitt, M.C., et al., The association of radiographically detected vertebral fractures with back pain and function: A prospective study. Annals of Internal Medicine, 1998. 128(10): p. 793-+.

12. Delmas, P.D., et al., Underdiagnosis of vertebral fractures is a worldwide problem: The IMPACT study. Journal of Bone and Mineral Research, 2005. 20(4): p. 557-563.

13. Kaptoge, S., et al., When should the doctor order a spine X-ray? Identifying vertebral fractures for 
osteoporosis care: results from the European Prospective Osteoporosis Study (EPOS). J Bone Miner Res, 2004. 19(12): p. 1982-93.

14. Ross, P.D., Clinical consequences of vertebral fractures. Am J Med, 1997. 103(2A): p. 30S-42S; discussion 42S-43S.

15. Kaptoge, S., et al., Whom to treat? The contribution of vertebral X-rays to risk-based algorithms for fracture prediction. Results from the European Prospective Osteoporosis Study. Osteoporos Int, 2006. 17(9): p. 136981.

16. Christiansen, B.A. and M.L. Bouxsein, Biomechanics of vertebral fractures and the vertebral fracture cascade. Curr Osteoporos Rep, 2010. 8(4): p. 198-204.

17. Delmas, P.D., et al., Severity of prevalent vertebral fractures and the risk of subsequent vertebral and nonvertebral fractures: results from the MORE trial. Bone, 2003. 33(4): p. 522-532.

18. Ismail, A.A., et al., Prevalent vertebral deformity predicts incident hip though not distal forearm fracture: results from the European Prospective Osteoporosis Study. Osteoporos Int, 2001. 12(2): p. 85-90.

19. Lee, T.C.M., P.E.; O’Brien, F.J.; O’Mahoney, D., Taylor, D.; Bruzzi, M.; Rackard, S.M.; Kennedy, O.D.; Mahony, N.J.; Harrison, N.; Lohfield, S.; Brennan, O.; Gleeson, J.; Hazenberg, J.G.; Mullins, L.; Tyndyk, M.; McNamara, L.M.; O’Kelly, K.U.; Prendergast, P.J. , Bone for life: osteoporosis, bone remodelling and computer simulation., in Topics in Bio-Mechanical Engineering. 2004, Prendergast, P.J. and McHugh, P.E. Eds: Trinity Centre for Bio-Engineering \& National Centre for Biomedical Engineering Science, Dublin, Ireland. . p. 5893.

20. Wustrack, R., et al., Predictors of new and severe vertebral fractures: results from the HORIZON Pivotal Fracture Trial. Osteoporos Int, 2012. 23(1): p. 53-8.

21. Briggs, A.M., et al., The effect of osteoporotic vertebral fracture on predicted spinal loads in vivo. Eur Spine J, 2006. 15(12): p. 1785-95.

22. Kanis, J.A., et al., A new approach to the development of assessment guidelines for osteoporosis. Osteoporos Int, 2002. 13(7): p. 527-36.

23. Kanis, J.A., et al., The use of clinical risk factors enhances the performance of BMD in the prediction of hip and osteoporotic fractures in men and women. Osteoporosis International, 2007. 18(8): p. 1033-1046.

24. Kanis, J.A., et al., FRAX (TM) and the assessment of fracture probability in men and women from the UK. Osteoporosis International, 2008. 19(4): p. 385-397.

25. Chapurlat, R., Contribution and limitations of the FRAX (R) tool. Joint Bone Spine, 2013. 80(4): p. 355357.
26. Ensrud, K.E., et al., A Comparison of Prediction Models for Fractures in Older Women Is More Better? Archives of Internal Medicine, 2009. 169(22): p. 20872094.

27. Rubin, K.H., et al., Comparison of different screening tools (FRAX(R), OST, ORAI, OSIRIS, SCORE and age alone) to identify women with increased risk of fracture. A population-based prospective study. Bone, 2013. 56(1): p. 16-22.

28. van Geel, T.A., et al., Individualizing fracture risk prediction. Maturitas, 2010. 65(2): p. 143-8.

29. Langsetmo, L., et al., Using the Same Bone Density Reference Database for Men and Women Provides a Simpler Estimation of Fracture Risk. Journal of Bone and Mineral Research, 2010. 25(10): p. 2108-2114.

30. Moayyeri, A., et al., Is QUS or DXA Better for Predicting the 10-Year Absolute Risk of Fracture? Journal of Bone and Mineral Research, 2009. 24(7): p. 13191325.

31. Masi, L., Epidemiology of osteoporosis. Clin Cases Miner Bone Metab, 2008. 5(1): p. 11-3.

32. Boehm, H.F., et al., Prediction of the fracture load of whole proximal femur specimens by topological analysis of the mineral distribution in DXA-scan images. Bone, 2008. 43(5): p. 826-31.

33. Gregg, E.W., et al., The epidemiology of quantitative ultrasound: a review of the relationships with bone mass, osteoporosis and fracture risk. Osteoporos Int, 1997. 7(2): p. 89-99.

34. Schechner, Z., et al., A poisson process model for hip fracture risk. Med Biol Eng Comput, 2010. 48(8): p. 799810.

35. Sornay-Rendu, E., et al., How to predict fragility fracture beyond 10 years? The OFELY study. J Clin Endocrinol Metab, 2014. 99(12): p. 4690-7.

36. Kanis, J.A., et al., European guidance for the diagnosis and management of osteoporosis in postmenopausal women. Osteoporosis International, 2013. 24(1): p. 23-57.

37. Meunier, P.J., et al., The effects of strontium ranelate on the risk of vertebral fracture in women with postmenopausal osteoporosis. New England Journal of Medicine, 2004. 350(5): p. 459-468.

38. Reginster, J.Y., et al., Strontium ranelate reduces the risk of nonvertebral fractures in postmenopausal women with osteoporosis: Treatment of Peripheral Osteoporosis (TROPOS) study. J Clin Endocrinol Metab, 2005. 90(5): p. 2816-22.

39. Boccaccio, A., et al., Finite element analysis of cancellous bone failure in the vertebral body of healthy and osteoporotic subjects. Proc Inst Mech Eng H, 2008. 222(7): p. 1023-36. 
40. Zhang, L., et al., The biomechanical effects of osteoporosis vertebral augmentation with cancellous bone granules or bone cement on treated and adjacent nontreated vertebral bodies: a finite element evaluation. Clin Biomech (Bristol, Avon), 2010. 25(2): p. 166-72.

41. MacNeil, J.A.M., et al., Predicting fracture using 2D finite element modelling. Medical Engineering \& Physics, 2012. 34(4): p. 478-484.

42. Kaneko, M., et al., Prediction of Proximal Femur Strength by a Quantitative Computed Tomography-Based Finite Element Method-Creation of Predicted Strength Data of the Proximal Femur According to Age Range in a Normal Population and Analysis of Risk Factors for Hip Fracture. Osteoporosis International, 2011. 22: p. 123124.

43. Bryan, R., P.B. Nair, and M. Taylor, Use of a statistical model of the whole femur in a large scale, multi-model study of femoral neck fracture risk. Journal of Biomechanics, 2009. 42(13): p. 2171-2176.

44. Bessho, M., et al., Prediction of proximal femur strength using a CT-based nonlinear finite element method: Differences in predicted fracture load and site with changing load and boundary conditions. Bone, 2009. 45(2): p. 226-231.

45. Derikx, L.C., et al., Implementation of asymmetric yielding in case-specific finite element models improves the prediction of femoral fractures. Computer Methods in Biomechanics and Biomedical Engineering, 2011. 14(2): p. 183-193.

46. Tellache, M.P., M.; Chabrand, P.; Hochard, C. , Femoral neck fracture prediction by anisotropic yield criteria. European Journal of Computational Mechanics, 2009. 18: p. 33-41.

47. Amin, S., et al., Association of hip strength estimates by finite-element analysis with fractures in women and men. J Bone Miner Res, 2011. 26(7): p. 1593-600.

48. Keaveny, T.M., et al., Femoral Bone Strength and Its Relation to Cortical and Trabecular Changes After Treatment With PTH, Alendronate, and Their Combination as Assessed by Finite Element Analysis of Quantitative CT Scans. Journal of Bone and Mineral Research, 2008. 23(12): p. 1974-1982.

49. Lopez, E., et al., A mechanical model for predicting the probability of osteoporotic hip fractures based in DXA measurements and finite element simulation. Biomedical Engineering Online, 2012. 11.

50. Mazess, R.B.B., H. , Bone density of the spine and femur in adult white females. . Calcified Tissue International, 1999. 65: p. 91-99.

51. Miller, P.D., et al., Efficacy of monthly oral ibandronate is sustained over 5 years: the MOBILE longterm extension study. Osteoporosis International, 2012. 23(6): p. 1747-1756.
52. Bianchi, G., et al., Long-term administration of quarterly IV ibandronate is effective and well tolerated in postmenopausal osteoporosis: 5-year data from the DIVA study long-term extension. Osteoporos Int, 2012. 23(6): p. 1769-78.

53. Reginster, J.Y., et al., Long-term treatment of postmenopausal osteoporosis with strontium ranelate: results at 8 years. Bone, 2009. 45(6): p. 1059-64.

54. Black, D.M., et al., The effect of 3 versus 6 years of zoledronic acid treatment of osteoporosis: a randomized extension to the HORIZON-Pivotal Fracture Trial (PFT). J Bone Miner Res, 2012. 27(2): p. 243-54.

55. Bone, H.G., et al., The effect of three or six years of denosumab exposure in women with postmenopausal osteoporosis: results from the FREEDOM extension. J Clin Endocrinol Metab, 2013. 98(11): p. 4483-92.

56. Felsenberg, D., et al., Efficacy of Monthly Oral Ibandronate Is Maintained over 5 Years: The Mobile Lte Study. Osteoporosis International, 2009. 20: p. 15-15.

57. Brennan, T.C., et al., Strontium ranelate effects in human osteoblasts support its uncoupling effect on bone formation and bone resorption. Journal of Bone and Mineral Research, 2007. 22: p. S139-S139.

58. Brennan, T.C., et al., Osteoblasts play key roles in the mechanisms of action of strontium ranelate. $\mathrm{Br} \mathrm{J}$ Pharmacol, 2009. 157(7): p. 1291-300.

59. Baron, R. and Y. Tsouderos, In vitro effects of S12911-2 on osteoclast function and bone marrow macrophage differentiation. Eur J Pharmacol, 2002. 450(1): p. 11-7.

60. Ammann, P., et al., Strontium ranelate improves bone resistance by increasing bone mass and improving architecture in intact female rats. J Bone Miner Res, 2004. 19(12): p. 2012-20.

61. Nielsen, S.P., et al., Influence of strontium on bone mineral density and bone mineral content measurements by dual X-ray absorptiometry. Journal of Clinical Densitometry, 1999. 2(4): p. 371-379.

62. Papapoulos, S., et al., Five years of denosumab exposure in women with postmenopausal osteoporosis: Results from the first two years of the FREEDOM extension. Journal of Bone and Mineral Research, 2012. 27(3): p. 694-701.

63. Papapoulos, S., et al., The effect of 8 or 5 years of denosumab treatment in postmenopausal women with osteoporosis: results from the FREEDOM Extension study. Osteoporos Int, 2015. 26(12): p. 2773-83.

64. López, E.H., A.; Gracia, L. , Diseño y desarrollo de un modelo de predicción de fracturas osteoporóticas. Aplicación al Cuello Femoral y a la Columna Lumbar (in Spanish). 2010, Saarbrücken, Germany.). Lambert Academic Publishing. 
65. Carter, D.R. and W.C. Hayes, The compressive behavior of bone as a two-phase porous structure. J Bone Joint Surg Am, 1977. 59(7): p. 954-62.

66. Kargarnovin, M.H.B.-E., M.; Katoozian, H.R., Damage initiation and growth in a long bone under increasing monotonic loading using the continuum damage mechanics principle., in Fifth International Conference on Engineering Computational Technology. 2006: Las Palmas de Gran Canaria, Spain. .

67. Paris, P.E., F. A, A critical analysis of crack propagation laws. Transactions of the American Society of Mechanical Engineers 1963. 85(4): p. 528-534.

68. Taylor, D., Microcrack growth parameters for compact bone deduced from stiffness variations. J Biomech, 1998. 31(7): p. 587-92.

69. Denoziere, G. and D.N. Ku, Biomechanical comparison between fusion of two vertebrae and implantation of an artificial intervertebral disc. Journal of Biomechanics, 2006. 39(4): p. 766-775.

70. Ibarz, E., et al., Development and kinematic verification of a finite element model for the lumbar spine: application to disc degeneration. Biomed Res Int, 2013. 2013: p. 705185.

71. Siemens, I-deas ${ }^{\circledR} 11$ NX Series PLM software [http://www.plm.automation.siemens.com /] 2013: Plano (Texas).

72. White, A.A.P., M.M. (1990). Clinical Biomechanics of the Spine 2nd ed, ed. Lippincott Williams \& Wilkins. 1990, Philadelphia, Pensilvania, USA.

73. Kapandji, I.A., The Physiology of the Joints 6th ed, ed. C. Livingstone. 2008, New York, USA.
74. Dassault system's simulia corp, U., The ABAQUS 6.12 ® [http://www.3ds.com/] 2013.

75. Dall'Ara, E., et al., QCT-based finite element models predict human vertebral strength in vitro significantly better than simulated DEXA. Osteoporosis International, 2012. 23(2): p. 563-572.

76. Lindsay, R., et al., Risk of new vertebral fracture in the year following a fracture. Jama, 2001. 285(3): p. 3203.

77. Baker-LePain, J.C., et al., Active shape modeling of the hip in the prediction of incident hip fracture. $\mathrm{J}$ Bone Miner Res, 2011. 26(3): p. 468-74.

78. Nasto, L.A., et al., Clinical predictors of vertebral osteoporotic fractures in post-menopausal women: a cross-sectional analysis. European Review for Medical and Pharmacological Sciences, 2012. 16(9): p. 12271234.

79. Kanis, J.A., et al., Assessment of fracture risk. Osteoporos Int, 2005. 16(6): p. 581-9.

80. Lopez, E., et al., Application of a model based on dual-energy X-ray absorptiometry and finite element simulation for predicting the probability of osteoporotic hip fractures to a sample of people over 60 years. Proc Inst Mech Eng H, 2015. 229(5): p. 369-85.

81. Chevalier, Y., et al., A patient-specific finite element methodology to predict damage accumulation in vertebral bodies under axial compression, sagittal flexion and combined loads. Comput Methods Biomech Biomed Engin, 2008. 11(5): p. 477-87.

82. Yang, H., et al., Effective modulus of the human intervertebral disc and its effect on vertebral bone stress. J Biomech, 2016. 